\title{
On uncertainty of Jupiter's core mass due to observational errors
}

\author{
Yasunori Hori ${ }^{1}$, Takayoshi Sano ${ }^{2}$, Masahiro Ikoma ${ }^{1}$ and Shigeru Ida ${ }^{1}$ \\ ${ }^{1}$ Department of Earth and Planetary Sciences, Tokyo Institute of Technology, \\ Ookayama, Meguro-ku, Tokyo 152-8551, Japan \\ email: hori@geo.titech.ac.jp \\ ${ }^{2}$ Institute of Laser Engineering, Osaka University, \\ Yamadaoka, Suita, Osaka 565-0871, Japan
}

\begin{abstract}
The origins of extrasolar gas giant planets have been discussed, based on our understanding of the gas giant planets in the solar system, Jupiter and Saturn. However, how Jupiter and Saturn formed is still uncertain because of the uncertainty in their interiors, especially the core mass $\left(M_{\mathrm{c}}\right)$. The uncertainty in $M_{\mathrm{c}}$ is partly due to those in observational data such as gravitational moments $\left(J_{2 n}\right)$, equatorial radius $\left(R_{\mathrm{eq}}\right)$ and 1-bar temperatures $\left(T_{1 \mathrm{bar}}\right)$. New frontiers mission to Jupiter by NASA (JUNO) launched in 2011 is expected to reduce the observational errors. However, it is not necessarily clear yet which observational uncertainty dominates and how accurate observation is needed to constrain $M_{\mathrm{c}}$ enough to know the origin of Jupiter. Thus, modeling the interior of Jupiter, we evaluate each effect on $M_{\mathrm{c}}$ and required precision. We have found that the observational error of $5 \%$ in $T_{1 \text { bar }}$ yields an error of several $M_{\oplus}$ in $M_{c}$. We have also found that the values of $J_{6}$ of our successful models are confined in a narrow range compared to its observational error. This implies that comparison between the values of $J_{6}$ of our successful models and the $J_{6}$ value obtained from JUNO mission helps us to know whether the present theoretical model is valid.
\end{abstract}

Keywords. gas giant planet, interior structure, core mass, gravitational moment

\section{Introduction}

To date, more than 250 exoplanets have been detected (e.g., Butler et al. 2006). The estimated planetary masses suggest that at least $90 \%$ of them should be gas giant planets. The interior structure, formation and evolution of those planets have been discussed, based on our knowledge of the gas giant giants, Jupiter and Saturn, in our solar system.

However, the interior structures of Jupiter and Saturn, especially the masses of their cores $\left(M_{\mathrm{c}}\right)$, are still uncertain; thus it is not yet possible to determine how they formed. The interior structure of the gas giant planets is determined by finding theoretically proper models that satisfy observational constraints such as equatorial radii $\left(R_{\text {eq }}\right)$, 1bartemperatures $\left(T_{1 \text { bar }}\right)$ and even gravitational moments $\left(J_{2 n}\right)$. Errors of the observational data and uncertainty in equation of state (EOS) for hydrogen and helium result in the uncertainty in $M_{\mathrm{c}}$ : Estimated core masses of Jupiter and Saturn are 0 to $14 M_{\oplus}$ and 15 to $25 M_{\oplus}$, respectively (Saumon \& Guillot 2004). On the other hand, the core accretion model, which is a widespread scenario for gas giant formation, requires cores larger than about $10 M_{\oplus}$ as seeds to form gas giant planets (e.g., Pollack et al. 1996). Thus, the prediction by theories of the interior structure, especially of Jupiter, is not consistent with that by formation theories. We need to limit the possible range of $M_{\mathrm{c}}$ more tightly by reducing the uncertainties in EOS and observational data.

Fortunately, more precise observational constraints will be obtained in the near future by JUNO launched in 2011, while it might not be easy to reduce the uncertainties in EOS 
soon. However, it is not necessarily clear in the literature which observational uncertainty dominates and how accurate observation is required to constrain $M_{\mathrm{c}}$ more tightly. For example, the results by Saumon \& Guillot (2004) include all of the uncertain factors. Therefore, in this study, we evaluate each effect on $M_{\mathrm{c}}$ of Jupiter and required observation precision by focusing on errors of the observational data.

\section{Modeling}

We model the static structure of gas giant planets in a similar way to previous studies (e.g., CEPAM; Guillot \& Morel 1995). We assume that the interior is three-layered, namely, a icy/rocky core, metallic-hydrogen and molecular-hydrogen layers outward; each layer is chemically homogeneous. The interior is assumed to be fully convective (i.e. isoentropic). We also assume that the planetary rotation is rigid and axisymmetrical. The equations of state used in this study are Saumon \& Chabrier EOS (Saumon et al. 1995) for hydrogen and helium and Hubbard \& Marley's (1989) EOS for ice and rock.

The input parameters and their ranges of values are listed in Table 1 . Those include 1-bar temperature $\left(T_{1 \mathrm{bar}}\right)$, the mass fraction of elements heavier than hydrogen in the molecular-hydrogen layer $\left(Y_{z}^{\text {mole }}\right)$, and the ratio of ice to rock in the core $\left(f_{\text {ice }}\right)$. The range of $T_{1 \text { bar }}$ corresponds to the current observational errors (see Table 2 ). We assume large $Y_{z}$ mole because in situ measurements by the Galileo probe suggest the heavy elements are enriched in the atmosphere of Jupiter compared to the solar abundance. In our calculations, $f_{\text {ice }}$ is fixed at 0.5 , because the effects of observational errors are of special interest.

For a given set of values of the parameters, we integrate the interior structure. Our successful models are such that the values of $R_{\text {eq }}, J_{2}, J_{4}$ and $J_{6}$ are within their observational errors given on Table 2. Changing values of $M_{\mathrm{c}}$ and the fraction of heavy elements (including helium) in the metallic layer $\left(Y_{z}^{\text {metal }}\right)$, we look for successful models in an iterative way.

Table 1. Input parameters (Guillot 1999)

\begin{tabular}{ccc}
\hline$T_{1 \text { bar }}[\mathrm{K}]$ & $Y_{z}^{\text {mole }}$ & $f_{\text {ice }}$ \\
\hline $160-170$ & $0.345-0.380$ & 0.5 \\
\hline
\end{tabular}

Table 2. Observational data (Guillot 2005)

\begin{tabular}{cccccc}
\hline & $T_{\text {bar }}[\mathrm{K}]$ & $J_{2} \times 10^{2}$ & $J_{4} \times 10^{4}$ & $J_{6} \times 10^{4}$ & $R_{\mathrm{eq}} \times 10^{9}[\mathrm{~cm}]$ \\
\hline Jupiter & $165 \pm 5$ & $1.4697 \pm 0.0001$ & $-5.84 \pm 0.05$ & $0.31 \pm 0.20$ & $7.1492 \pm 0.0006$ \\
Saturn & $135 \pm 5$ & $1.6332 \pm 0.0010$ & $-9.19 \pm 0.40$ & $1.04 \pm 0.50$ & $5.8210 \pm 0.0006$ \\
\hline
\end{tabular}

\section{Results}

Figure 1 plots $M_{\mathrm{c}}$ and the total mass of heavy elements (i.e., elements other than hydrogen and helium) contained in the envelope $\left(M_{z}\right)$ of successful interior models. We calculated $M_{z}$ by using $Y_{z}^{\text {mole }}$ and $Y_{z}^{\text {metal }}$ under the constraint that the total mass fraction of helium of Jupiter must equal to that of the protosolar disk. The domain enclosed with dotted lines in Fig.1 contains all the successful models. Each small box 


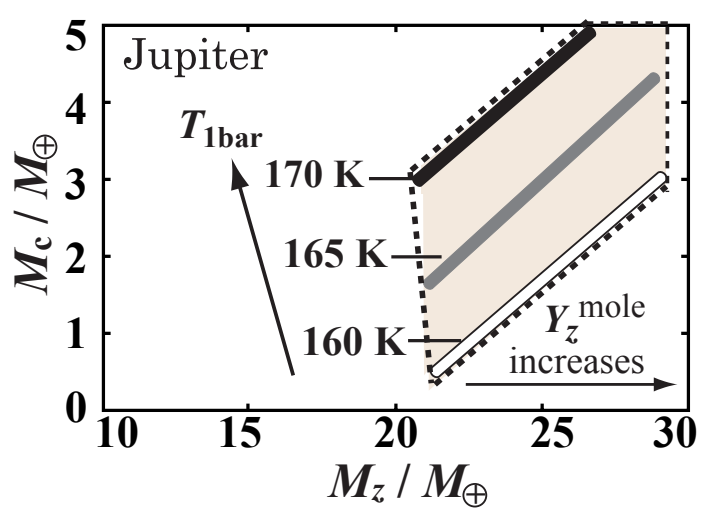

Figure 1. Possible ranges of the core mass $\left(M_{\mathrm{c}}\right)$ and the total mass of heavy elements contained in the envelope $\left(M_{z}\right)$ of Jupiter that match the observed values, $R_{\text {eq }}, J_{2}, J_{4}$ and $J_{6}$ within $1 \sigma$.

corresponds to the case of a fixed value of $T_{1 \mathrm{bar}}$; the black, gray, and white boxes represent results for $170 \mathrm{~K}, 165 \mathrm{~K}$, and $160 \mathrm{~K}$ respectively. The left end of each box corresponds to the result for $Y_{z}{ }^{\text {mole }}=0.345$ and $Y_{z}^{\text {mole }}$ increases from left to right. Note that all the core masses of our successful models are less than $10 M_{\oplus}$, which do not always mean that $M_{\mathrm{c}}$ of Jupiter could be less than $10 M_{\oplus}$. Those results arise because our calculations adopt only specific EOS models and a limited range of $Y_{z}{ }^{\text {mole }}$. That is enough to see the sensitivity of $M_{\mathrm{c}}$ to the observational constraints.

In Fig. 1, one finds that the error of $5 \%$ in yields an error of several $M_{\oplus}$ in $M_{c}$. Higher $T_{1 \text { bar }}$ results in larger $M_{\mathrm{c}}$ because the entropy of the interior is also high, which causes a decrease in the interior density. This reduces the degree of concentration near the deep part. As a result, higher $T_{1 \text { bar }}$ eventually yields larger $M_{c}$ to satisfy the constraint of $J_{2}$, namely, the inertia moment. The difference in $M_{z}$ caused by that in $T_{1 \text { bar }}$ is so small that it would be of little significance from the viewpoint of planet formation.

The uncertainty in $Y_{z}^{\text {mole }}$ also yields an error of a few $M_{\oplus}$ in $M_{\mathrm{c}}$. An increase in $Y_{z}{ }^{\text {mole }}$ from 0.345 to 0.380 is found to result in a increase in $M_{z}$ by about $10 M_{\oplus}$. Then, larger $M_{z}$ reduces the degree of concentration near the deep part like the case of higher $T_{1 \mathrm{bar}}$. Larger $M_{\mathrm{c}}$ is thus required to satisfy the constraint of $J_{2}$.

Finally, we have found two implications concerning $J_{4}$ and $J_{6}$ for the interior structure of Jupiter. The uncertainty in $M_{\mathrm{c}}$ for fixed values of $T_{1 \mathrm{bar}}$ and $Y_{z}{ }^{\text {mole }}$ is due to those in observational constraints, especially $J_{4}$ and $J_{6}$. That uncertainty is small compared to the one due to the errors of $T_{1 \mathrm{bar}}$ and $Y_{z}{ }^{\text {mole }}$. Our calculations suggest that the interior models that match the observed $J_{4}$ require a little difference between $Y_{z}^{\text {mole }}$ and $Y_{z}{ }^{\text {metal }}$. Also, we have found that the values of $J_{6}$ in our successful models are in a narrow area from $0.32 \times 10^{-4}$ to $0.37 \times 10^{-4}$ compared to the observational error of $J_{6}$.

\section{Conclusions}

We have realized that the error in $T_{1 \text { bar }}$ should be responsible for the uncertainty in $M_{\mathrm{c}}$ mostly. To avoid the uncertainty of several or a few Earth-mass in $M_{\mathrm{c}}$, the error in $T_{1 \text { bar }}$ is reduced by less than $1 \%$ at least.

Furthermore, the importance of the uncertainty in $T_{1 \text { bar }}$ suggests that the thermal structure must be investigated in more detail. Although our calculations assumed that the interior is fully convective, there exists one possibility that the interior is superadiabatic. In fact, Guillot et al. (2004) estimated temperature gradient based on the 
mixing theory and found that this possibility may be high. The resultant hot interior would yield larger $M_{\mathrm{c}}$ like in the case of higher $T_{1 \mathrm{bar}}$, which has the potential to solve the formation problem of Jupiter, i.e. the problem of the possibility that Jupiter may have a small core or be a coreless planet.

We have also reconfirmed the need for accurate data for $J_{4}$ and $J_{6}$. Our successful models prefer a little difference between $Y_{z}^{\text {mole }}$ and $Y_{z}{ }^{\text {metal }}$ to satisfy the $J_{4}$ constraint. This suggests that $J_{4}$ is a key to know the composition of the metallic layer (i.e. $Y_{z}{ }^{\text {metal }}$ ) that probes are unable to reach. In other words, if we obtain the accurate $J_{4}$ and $Y_{z}$ mole of Jupiter by in situ measurements such as JUNO, we will know the content of heavy elements of Jupiter. We also found that the resultant $J_{6}$ values are confined in a narrow range compared to its observational error. This implies that whether the $J_{6}$ value obtained by JUNO is in the narrow range predicted by interior models or not helps us to confirm the validity of the present interior model of Jupiter. Finally, the error of the observed $J_{6}$ is required to be within the narrow range at least.

\section{References}

Butler R. P., Wright, J. T., Marcy, G. W., Fischer, D. A., Vogt, S. S., Tinney, C. G., Jones, H. R. A., Carter, B. D., Johnson, J. A., McCarthy, C. \& Penny, A. J. 2006, ApJ, 646, 505

Guillot, T. 1999, Plan. Space. Sci. 47, 1183

Guillot, T. 2005, Ann. Rev. Earth Planet. Sci., 33, 493

Guillot, T. \& Morel, P. 1995, ApJS, 109, 109

Guillot, T., Stevenson, D. J., Hubbard, W. B. \& Saumon, D. 2004, F. Bagenal et al. ed., Cambridge University Press

Hubbard, W. B. \& Marley, M. S. 1989, Icarus, 78, 102

Pollack, J. B., Hubickyj, O., Bodenheimer, P., Lissauer, J. J., Podolak, M. \& Greenzweig, Y. 1996, Icarus, 124, 62

Saumon, D., Chabrier, G., \& Van Horn, H. M. 1995, ApJS, 99, 713

Saumon, D. \& Guillot, T. 2004, ApJ, 609, 1170 